\title{
Estudo da viabilidade econômica da irrigação da pupunheira (Bactris Gasipaes H.B.K.) para Ilha Solteira - SP, Brasil ${ }^{1}$
}

\author{
Economic viability study of the pupunha crop (Bactris Gasipaes \\ H.B.K.) irrigation for Ilha Solteira - SP, Brazil
}

Patrícia Angélica Alves Marques ${ }^{2}$ Rubens Duarte Coelho ${ }^{3}$

\section{RESUMO}

No presente trabalho avaliou-se a viabilidade econômica da irrigação da pupunheira em Ilha Solteira, para as características locais através do programa Pupunha, que permite a variação dos fatores que interferem no custo da irrigação, fornecendo uma família de curvas comparativas para diversas situações. Estudou-se o efeito do custo da água associado a lâmina de irrigação e a sensibilidade dos equipamentos de irrigação localizada devido a variação da vida útil, associado ao tipo de motor utilizado. Usando a produtividade de palmito de sequeiro de $700 \mathrm{~kg} \mathrm{ha}^{-1}$ e a produtividade do palmito irrigado de $2500 \mathrm{~kg} \mathrm{ha}^{-1}$, concluiuse que para todas as simulações realizadas, a irrigação da pupunheira é viável para a localidade de Ilha Solteira - SP, pois o aumento de produtividade real de $257 \%$, ocorrido com a irrigação, supera o máximo "acréscimo de produtividade necessário" de 130,33\%, proporcionando lucro ao produtor.

Palavras-chave: estudo econômico, palmito, viabilidade.

\section{ABSTRACT}

The objective of this study was to evaluate the economic viability of the irrigation of the Pupunha crop in Ilha Solteira, São Paulo, Brazil for the local parameters. Through the program Pupunha, being obtained a family of comparative curves for several situations. The effect of the cost of the water was studied associated with the irrigation levels. The sensitivity of the equipments due the variation of the life useful was studied associate to the type of used motor. Using the yield of the no-irrigate palm heart of $700 \mathrm{~kg} \mathrm{ha}^{-1}$ and yield of the irrigated palm heart of $2500 \mathrm{~kg} \mathrm{ha}^{-1}$, for all the accomplished simulations, the income of the production increment overcomes the cost of the irrigation, supplying profit to the producer.

Key words: economical study, palm heart, viability.

\section{INTRODUÇÃo}

A pupunheira é uma palmeira cespitosa que pode atingir 20 metros de altura. O sistema radicular é fasciculado e superficial, característica que leva a planta a apresentar baixa resistência à seca. A pupunheira apresenta vários usos, tais como: consumo humano direto de seus frutos, farinha para panificação, farinha para ração animal e palmito. O palmito é hoje o produto econômico mais importante obtido da pupunha, sendo comercializado na forma de toletes de palmito em conserva (palmito de primeira), pedaços e rodelas (TONET et al., 1999; CLEMENT, 2000). De acordo com FERREIRA (1987) e TONET et al. (1999), a pupunheira é originária de regiões tropicais com alta precipitação pluviométrica e solos de baixa fertilidade. Entretanto está adaptada em diferentes condições ecológicas nos trópicos. É encontrada desde o nível do mar até próximo dos $2.000 \mathrm{~m}$ de altitude, mas acima de $700 \mathrm{~m}$ de altitude produz pouco.

${ }^{1}$ Trabalho desenvolvido no Curso de Pós-graduação em Irrigação e Drenagem da ESALQ/USP em 1999.

${ }^{2}$ Engenheiro Agrônomo Doutoranda do Depto. de Engenharia Rural ESALQ/USP, Curso de Pós-graduação em Irrigação e Drenagem. Av. dos Marins, 100, bloco 54, apto 11, glebas Califórnia, 13403-025 Piracicaba, SP. E-mail: paamarqu@esalq.usp.br Autor para correspondência.

3Professor, Doutor, Depto. Engenharia Rural, ESALQ/USP. 
A pupunheira é uma planta tropical muito exigente em água. Para regiões com mais de dois meses seguidos de déficit hídrico, a irrigação torna-se necessária. Diversos trabalhos têm demonstrado que a produção de palmito acompanha a distribuição pluviométrica. Conseqüentemente, locais que apresentam um período seco prolongado terão sua produção prejudicada. Dentre os fatores limitantes à produção do palmito, o déficit hídrico é um fator contornável, uma vez que se pode dispor de um sistema de irrigação (MORO, 1996).

Nas regiões onde há insuficiência ou má distribuição das chuvas, em alguns períodos do ano, torna-se inviável a exploração agrícola econômica; daí por que a irrigação justifica-se como recurso tecnológico indispensável ao aumento da produtividade das culturas. Entretanto a viabilidade econômica é um fator indispensável para sua adoção pelos agricultores (FRIZZONE et al., 1994). A agricultura irrigada exige alto investimento em obras e aquisição de equipamentos, transporte, controle e distribuição de água; além de gastos com energia e mão-de-obra para operação do sistema, que representam importantes custos adicionais, os quais devem ser pagos pelo incremento de produtividade proporcionado pelo fornecimento de água às plantas (AZEVEDO, 1983 e CLARK et al., 1993).

Estudando o manejo da irrigação da pupunheira em Ilha Solteira, HERNANDEZ (2001a) observou que, sem irrigação, o número de plantas mortas foi alto, e o início da produção ficou postergado, significando prejuízo duplo, pois se perde o dinheiro investido na muda e em seu plantio, e por outro lado, fica-se sem receita, pois retarda a colheita inicial. $\mathrm{Na}$ sequência desta pesquisa HERNANDEZ (2001b) estudou a produtividade da pupuheira sem irrigação e com irrigação em diferentes frações da evaporação do tanque classe A (ECA), onde observou valores de produtividade para a pupunheira sem irrigação de $700 \mathrm{~kg} \mathrm{ha}^{-1}$ e para a pupunheira irrigada, com o uso de 1,0 ECA, uma produtividade de $2.500 \mathrm{~kg} \mathrm{ha}^{-1}$.

No oeste do Estado de São Paulo, áreas com irrigação obtiveram a primeira colheita aos 20 meses após a cultura instalada, enquanto que, sem irrigação, obtiveram a primeira colheita aos 30 - 36 meses (TONET et al., 1999). Especificamente para o Noroeste Paulista, de acordo com BOVI (1997), as exigências de temperatura para a pupunheira são atendidas, porém as chuvas deverão ser complementadas pelas irrigações. Ilha Solteira, Noroeste do Estado de São Paulo, assume particular importância para a agricultura do Estado, comenta HERNANDEZ et al. (1995), pois é uma das áreas com potencial para a irrigação, devido principalmente à formação dos represamentos provenientes da implantação do Complexo Hidroelétrico de Urubupungá. Em muitos locais, a má adaptação da pupunheira foi devida à falta de água, mas até o momento existem poucos experimentos sobre a irrigação da cultura. CLEMENT (1995) usou irrigação por sulcos e DEFRANK \& CLEMENT (1995) usaram aspersores acima do dossel do plantio, todos com bons resultados. Nenhum destes experimentos, no entanto, era planejado para avaliar a irrigação.

Neste trabalho, objetivou-se estudar a viabilidade econômica da irrigação da pupunheira, para as condições locais de Ilha Solteira, estudando-se o efeito do custo da água associado à lâmina de irrigação e a sensibilidade dos equipamentos à variação da vida útil associado ao tipo de motor utilizado.

\section{MATERIALEMÉTODOS}

Para o estudo da Viabilidade Econômica da Irrigação da pupunheira, utilizou-se o "Programa Pupunha" (MARQUES et al., 1999), que permite as combinações dos fatores reais do produtor, resultando em uma análise econômica mais específica e conseqüentemente mais ajustada à realidade das condições do local estudado, determinando a viabilidade da irrigação da cultura. O "Programa Pupunha" calcula o acréscimo de produtividade necessário para pagar os custos adicionais da irrigação, sendo permitida a variação dos principais fatores que interferem nestes referidos custos, obtendo-se então uma família de curvas que mostram a sensibilidade deste fator, para vários valores de produtividade de palmito de primeira $\left(\mathrm{kg} \mathrm{ha}^{-1}\right)$.

Para o primeiro caso, foram avaliados os efeitos da variação do custo da água $\left(\mathrm{R} \$ 0,00 \mathrm{~m}^{-3}\right.$, $\mathrm{R} \$ 0,03 \mathrm{~m}^{-3}$ e $\mathrm{R} \$ 0,05 \mathrm{~m}^{-3}$ ) em combinação com a lâmina de água aplicada, sendo uma aplicação de $100 \%$ da ETc referente a $4 \mathrm{~mm} \mathrm{dia}^{-1}$ e uma aplicação de $120 \%$ da ETc referente a $4,8 \mathrm{~mm} \mathrm{dia}^{-1}$. Um segundo estudo referiu-se à comparação entre os custos anuais utilizando o motor diesel e o motor elétrico, juntamente com o estudo da influência da diminuição da vida útil do equipamento. Os fatores fixos utilizados estão mostrados na tabela 1 .

O custo total de uma produção agrícola pode ser dividido em custos fixos e variáveis (MAROUELLI \& SILVA, 1998 e FRIZZONE, 1999). Os custos fixos são aqueles que ocorrem independente do número de horas anuais de operação do sistema de irrigação ( $\mathrm{R} \$$ ano $\left.^{-1} \mathrm{ha}^{-1}\right)$. Para obtê-los utilizou-se:

a) O Fator de Recuperação de Capital (FRC) utiliza o juros anuais e fornece um coeficiente que 
Tabela 1 - Fatores fixos utilizados no estudo da viabilidade econômica da irrigação da pupunheira na localidade de Ilha Solteira, Estado de São Paulo.

\begin{tabular}{|c|c|}
\hline Fatores & Valores \\
\hline Eto $\left(\mathrm{mm} \mathrm{dia}^{-1}\right)$ & 4,5 \\
\hline $\mathrm{Kc}$ & 0,85 (RAMOS, 1998) \\
\hline Etc $\left(\mathrm{mm} \mathrm{dia}^{-1}\right)$ & 4 \\
\hline Produtividade de sequeiro $\left(\mathrm{kg} \mathrm{ha}^{-1}\right)$ & 700 (LOPES et al., 2000 e HERNANDEZ, 2001b) \\
\hline Produtividade da pupunheira irrigada $\left(\mathrm{kg} \mathrm{ha}^{-1}\right)$ & 2500 (LOPES et al., 2000 e HERNANDEZ, 2001b) \\
\hline \multirow[t]{2}{*}{ Meses de déficit hídrico } & abril a setembro \\
\hline & (CENTURION, 1982 e HERNANDEZ et al., 1995) \\
\hline Lucratividade $(\%)$ & 80 \\
\hline Mão-de-obra (funcionário ha ${ }^{-1}$ ) & 0,5 \\
\hline Salário mensal (R\$) & 130,00 (AGRIANUAL, 1998) \\
\hline Preço do palmito de primeira $\left(\mathrm{R} \$ \mathrm{~kg}^{-1}\right)$ & 2,50 \\
\hline Horas de operação dia ${ }^{-1}$ & 20 \\
\hline Horas com desconto & 6 \\
\hline Dias de operação por mês & 30 \\
\hline Tarifa de consumo da Tarifa Verde $\left(\mathrm{R} \$ \mathrm{Kw}^{-1} \mathrm{~h}^{-1}\right)$ & 0,030 \\
\hline Tarifa de demanda da Tarifa Verde $\left(\mathrm{R} \$ \mathrm{Kw}^{-1}\right)$ & 3,32 \\
\hline Desconto para uso no horário das $23 \mathrm{~h}$ às $5 \mathrm{~h}(\%)$ & 70 \\
\hline Fator de potência & 0,92 \\
\hline Custo da rede elétrica instalada $\left(\mathrm{R} \$ . \mathrm{km}^{-1}\right)$ & 7000,00 \\
\hline Distância a ser instalada a rede elétrica $(\mathrm{Km})$ & 0,5 \\
\hline Vida útil da rede elétrica (anos) & 50 \\
\hline Juros de mercado (\% a.a.) & 12 \\
\hline Custo de manutenção (\%) & 3 \\
\hline Custo do óleo diesel $\left(\mathrm{R} \$\right.$ litro $\left.^{-1}\right)$ & 0,40 \\
\hline Consumo específico do motor diesel $\left(\mathrm{g} \mathrm{cv}^{-1} \mathrm{~h}^{-1}\right)$ & 170 \\
\hline Faixa de produtividade de palmito de sequeiro $\left(\mathrm{kg} \mathrm{ha}^{-1}\right)$ & 700 a 1700 \\
\hline Sistema de irrigação & microaspersão \\
\hline Custo de aquisição do equipamento de irrigação ( $\mathrm{R} \$$ ha $\left.^{-1}\right)$ & 4300,00 \\
\hline Vida útil do equipamento de irrigação (anos) & 15 \\
\hline Potência do motor $\left(\mathrm{cv} \mathrm{ha}^{-1}\right)$ & 1,3 \\
\hline Módulo de área de estudo (ha) & 1 \\
\hline Preço da água $\left(\mathrm{R} \$ \mathrm{~m}^{-3}\right)$ & 0,00 \\
\hline
\end{tabular}

permite, a partir do valor do investimento, calcular o custo fixo anual referente a este investimento (FRANCISCO, 1991; BERNARDO, 1995 e CAMARGO, 1998). Este cálculo leva em conta a vida útil do equipamento (anos) e a taxa de juros ao ano (\%) (equação 1).

$$
F R C=\frac{J \cdot(J+1)^{V}}{(J+1)^{V}-1}
$$

onde: FRC - fator de recuperação de capital; $\mathrm{J}$ - taxa de juros ao ano (\%); V - vida útil (anos).

b) Custo anual da Rede Elétrica (CE) leva em consideração a distância a ser instalada de rede elétrica, o custo da rede elétrica, a área do módulo e a vida útil do equipamento (equação 2).

$$
C E=\frac{D \cdot P R}{A \cdot V}
$$

onde: CE - custo anual da rede elétrica $\left(\mathrm{R} \$ \mathrm{ano}^{-1} \mathrm{ha}^{-1}\right) ; \mathrm{D}$ - distância da rede elétrica a ser instalada $(\mathrm{km}) ; \mathrm{PR}$ - preço da rede elétrica $\left(\mathrm{R} \$ \mathrm{~km}^{-1}\right)$; A - módulo de área (ha).

c) O Cálculo do custo fixo anual leva em conta o FRC, o CE e o preço de aquisição do equipamento ( $\mathrm{R} \$$ ha $\left.^{-1}\right)$ (equação 3).

$$
C F=F R C_{1} \cdot P S+F R C_{\text {rede }} \cdot C E
$$
onde: $\mathrm{CF}$ - custo fixo anual $\left(\mathrm{R} \$ \mathrm{ano}^{-1} \mathrm{ha}^{-1}\right)$; PS - preço de aquisição do equipamento de irrigação $\left(\mathrm{R} \$ \mathrm{ha}^{-1}\right) ; \mathrm{FRC}_{1}-\mathrm{FRC}$ aplicado a vida útil do equipamento de irrigação; $\mathrm{FRC}_{\text {rede }}-\mathrm{FRC}$ aplicado à vida útil da rede elétrica.

No cálculo dos custos variáveis anuais da irrigação, estão envolvidos os custos de energia, manutenção, mão de obra e custo da água (BERNARDO, 1995 e MAROUELLI \& SILVA, 1998). Segundo FRIZZONE (1999), o tipo de motor, elétrico ou a combustão é um parâmetro de grande efeito sobre os custos. Para o motor elétrico, é importante considerar a modalidade de tarifação elétrica bem como os custos com a linha de tensão se o ramal elétrico da concessionária estiver distante da estação de bombeamento. Segundo BERNARDO (1995), o custo de energia depende também, da potência absorvida do motor, do número de horas de trabalho e do consumo médio por hora. Neste trabalho, consideraram-se os motores elétrico e diesel, de acordo com os seguintes cálculos:

Ciência Rural, v. 33, n. 2, mar-abr, 2003. 
a1) O Custo de Energia do motor elétrico leva em conta a demanda e o consumo anual corrigidos pelo ICMS cobrado no estado (\%) (equações 4 e 5). $D a=((12-M) \cdot 10 \%+M) \cdot T d \cdot P \cdot 0,73259) \cdot(1+I C M S)$ onde: $\mathrm{Da}$ - demanda anual $\left(\mathrm{R} \$ \mathrm{ano}^{-1} \mathrm{ha}^{-1}\right)$; M - meses de operação do sistema de irrigação; Td tarifa de demanda $\left(\mathrm{R} \$ \mathrm{KW}^{-1}\right) ; \mathrm{P}$ - potência do motor $\left(\mathrm{cv} \mathrm{ha} \mathrm{a}^{-1}\right)$; ICMS - ICMS cobrado na região (\%). $C a=M \cdot(H d \cdot \cdot(1-D e)+H s) \cdot T c \cdot D o \cdot P \cdot 0,73259 \cdot(1+I C M S) \quad$ (5) onde: $\mathrm{Ca}-$ consumo anual $\left(\mathrm{R} \$ \mathrm{ano}^{-1} \mathrm{ha}^{-1}\right)$; Hd - horas com desconto (23h às 5h) (FRIZZONE, 1999); De - desconto da Portaria 105 DNAEE (\%); Hs - horas sem desconto; Tc - tarifa de consumo $\left(\mathrm{R} \$ \mathrm{KWh}^{-1}\right)$; Do - dias de operação por mês.

O custo anual da energia (equação 6) pode ser expresso pela soma do faturamento da demanda e o faturamento de consumo ajustados pelo fator de potência (FRIZZONE, 1999).

$$
\text { Cen }=(D a+C a) *\left(\frac{0,92}{\cos \varphi}\right)
$$

onde: Cen - custo de energia $\left(\mathrm{R} \$ \mathrm{ano}^{-1} \mathrm{ha}^{-1}\right)$; $\cos j$ - fator de potência.

a2) Custo de Energia do motor diesel leva em conta os meses de operação por ano, o número de dias irrigando por mês, o número de horas operando por mês, a potência do motor, o consumo específico do motor $\left(\mathrm{g} \mathrm{cv}^{-1} \mathrm{~h}^{-1}\right)$ e o preço do óleo diesel na propriedade (R\$ litro $\left.{ }^{-1}\right)$ (equação 7).

$$
\text { Cen }=C o \cdot P \cdot C s \cdot 0,00125 \cdot H \cdot D o \cdot M
$$

onde: Co - custo do óleo diesel na propriedade ( $\mathrm{R} \$$ litro $\left.^{-1}\right)$; $\mathrm{Cs}$ - consumo específico do motor diesel $\left(\mathrm{g} \mathrm{cV}^{-1} \mathrm{~h}^{-1}\right) ; \mathrm{H}$ - horas de operação por dia.

b) Custo de Manutenção leva em conta uma porcentagem do custo de Energia, esta porcentagem é um valor de entrada no programa (equação 8).

$$
C m=\frac{\% \cdot C e n}{100}
$$

onde: $\mathrm{Cm}$ - custo de manutenção $\left(\mathrm{R} \$\right.$ ano $\left.^{-1} \mathrm{ha}^{-1}\right)$.

c) Custo da Mão de Obra (equação 9) leva em consideração o salário médio pago, o número de funcionários, e os encargos pagos sobre o salário (MAROUELLI \& SILVA, 1998 e FRIZZONE, 1999).

$$
\begin{aligned}
& M O=S \cdot\left(1+\left(\frac{F+T+I+I T}{100}\right)\right) \cdot N f \quad \text { (9) } \\
& \text { onde: } M O \text { - custo da mão de obra }
\end{aligned}
$$
$\left(\mathrm{R} \$\right.$ ano $^{-1}$ ha $\left.^{-1}\right) ; \mathrm{S}$ - salário mensal $\left(\mathrm{R} \$\right.$ mês $\left.^{-1}\right) ; \mathrm{F}$ encargo pago relativo a férias em porcentagem do salário (\%); T - encargo pago relativo ao $13^{0}$ salário em porcentagem do salário (\%); I - encargo pago relativo ao INSS em porcentagem do salário (\%); IT - encargo pago relativo ao INSS do $13^{0}$ salário em porcentagem do salário (\%); $\mathrm{Nf}$-número de funcionários para o manejo da irrigação (homens ha-1). d) Custo da Água leva em conta a evapotranspiração da cultura, dias irrigando por mês, número de meses por ano e o preço da água $\left(\mathrm{R} \$ \mathrm{~m}^{-3}\right)$ (equação 10).

$$
\mathrm{Ca}=\mathrm{ETC} \cdot \mathrm{Do} \cdot \mathrm{M} \cdot \mathrm{Pa} \cdot 10
$$

onde: $\mathrm{Ca}$ - custo da água $\left(\mathrm{R} \$ \mathrm{ano}^{-1} \mathrm{ha}^{-1}\right)$; ETC - evapotranspiração da cultura $\left(\mathrm{mm} \mathrm{dia}^{-1}\right) ; \mathrm{Pa}-$ preço da água $\left(\mathrm{R} \$ \mathrm{~m}^{-3}\right)$. (equação 11).

e) Custo Total Variável Anual (CV)

$$
C V=C e n+C m+C a+M O
$$

onde: $\mathrm{CV}$ - custo variável anual $\left(\mathrm{R} \$ \mathrm{ano}^{-1}\right.$ $\left.\mathrm{ha}^{-1}\right)$.

O Custo total é a soma dos custos fixos anuais e dos custo variáveis anuais (FRIZZONE, 1999) visto na equação 12 .

$$
C T=C F+C V
$$

onde: CT - Custo Total anual $\left(\mathrm{R} \$ \mathrm{ano}^{-1} \mathrm{ha}^{-1}\right)$.

A receita bruta utilizada em uma análise financeira é obtida pela multiplicação da produtividade agrícola pelo preço pago (FRIZZONE, 1999). Assim a renda anual foi calculada considerando a produtividade de palmitos de primeira $\left(\mathrm{kg} \mathrm{ha}^{-1} \mathrm{ano}^{-1}\right)$ e o preço do palmito no mercado interno $\left(\mathrm{R} \$ \mathrm{~kg}^{-1}\right)$ (equação 13).

$$
R=P p \cdot \text { Ppal }
$$

onde: $\mathrm{R}$ - renda anual $\left(\mathrm{R} \$ \mathrm{ano}^{-1} \mathrm{ha}^{-1}\right) ; \mathrm{Pp}-$ produtividade de palmito de primeira $\left(\mathrm{kg} \mathrm{ha}^{-1} \mathrm{ano}^{-1}\right)$; Ppal - preço do palmito de primeira pago ao produtor $\left(\mathrm{R} \$ \mathrm{~kg}^{-1}\right)$.

O "Acréscimo de produtividade necessário", para que a renda advinda do incremento de produtividade superasse o custo da irrigação (equação 14), foi calculado baseado no Custo Total Anual da irrigação obtido e no acréscimo da Renda obtida para pagar este Custo Total. Para cada produtividade média versus o custo Total obteve-se um ponto, resultando numa curva para cada situação testada.

Acréscimo de Produtividade Necessário $=\left(\frac{C T}{R \cdot \frac{L}{100}}\right) \cdot 100(14)$

onde: L - Lucratividade esperada (\%). Após simular as combinações propostas, analisaram-se os casos de maior "acréscimo de produtividade necessário” (equação 14), os quais foram comparados com o incremento de produtividade real ocorrido na pupunheira irrigada em Ilha Solteira fornecida por LOPES et al. (2000) e HERNANDEZ (2001b), e assim sendo, determinou-se a viabilidade econômica da irrigação nesta localidade. 


\section{RESULTADOS E DISCUSSÃO}

Na figura 1 , considerou-se a ETc $(4 \mathrm{~mm}$ dia $\left.{ }^{1}\right)$ e uma aplicação de água em excesso, isto é, $120 \%$ da ETc $\left(4,8 \mathrm{~mm} \mathrm{dia}^{-1}\right)$ comparadas com três custos de água ( $\mathrm{R} \$ 0,00 \mathrm{~m}^{-3}, \mathrm{R} \$ 0,03 \mathrm{~m}^{-3}$ e $\mathrm{R} \$ 0,05 \mathrm{~m}^{-3}$ ), considerando motor elétrico. Pode ser observado que, com o aumento do custo da água, este excesso de $20 \%$ do volume de água aplicado resultou em um diferencial na variável “acréscimo de produtividade necessário”(equação 14). Por exemplo, considerando a curva de ETc de $4 \mathrm{~mm}$ dia ${ }^{1}$ e o máximo custo de água $\left(\mathrm{R} \$ 0,05 \mathrm{~m}^{-3}\right)$, na produtividade de palmito de sequeiro de $700 \mathrm{~kg} \mathrm{ha}^{-1} \mathrm{o}$ "acréscimo de produtividade necessário" foi de $115,93 \%\left(811,51 \mathrm{~kg} \mathrm{ha}^{-1}\right)$ e variando apenas para a curva de ETc de 4,8mm dia ${ }^{-1}$ com os demais fatores idênticos o "acréscimo de produtividade necessário" foi de $121,08 \%\left(847,56 \mathrm{~kg} \mathrm{ha}^{-1}\right)$. Portanto este excesso de água aplicado resultou em uma necessidade de aumento de produtividade de $36,05 \mathrm{~kg} \mathrm{ha}^{-1}$.

$\mathrm{Na}$ figura 2, comparou-se o uso de motor diesel e o uso de motor elétrico, considerando-se a vida útil do equipamento de irrigação igual a 5 anos, 10 anos e 15 anos. Utilizando-se, como exemplo, a produtividade de sequeiro de $700 \mathrm{~kg} \mathrm{ha}^{-1}$, observou-se que, para o motor diesel, com a variação da vida útil de 15 anos ("acréscimo de produtividade necessário" = $80,52 \%$ ) para 5 anos ("acréscimo de produtividade necessário" $=120,62 \%$ ) acarretou num diferencial de "acréscimo de produtividade necessário" de 40,1\%,

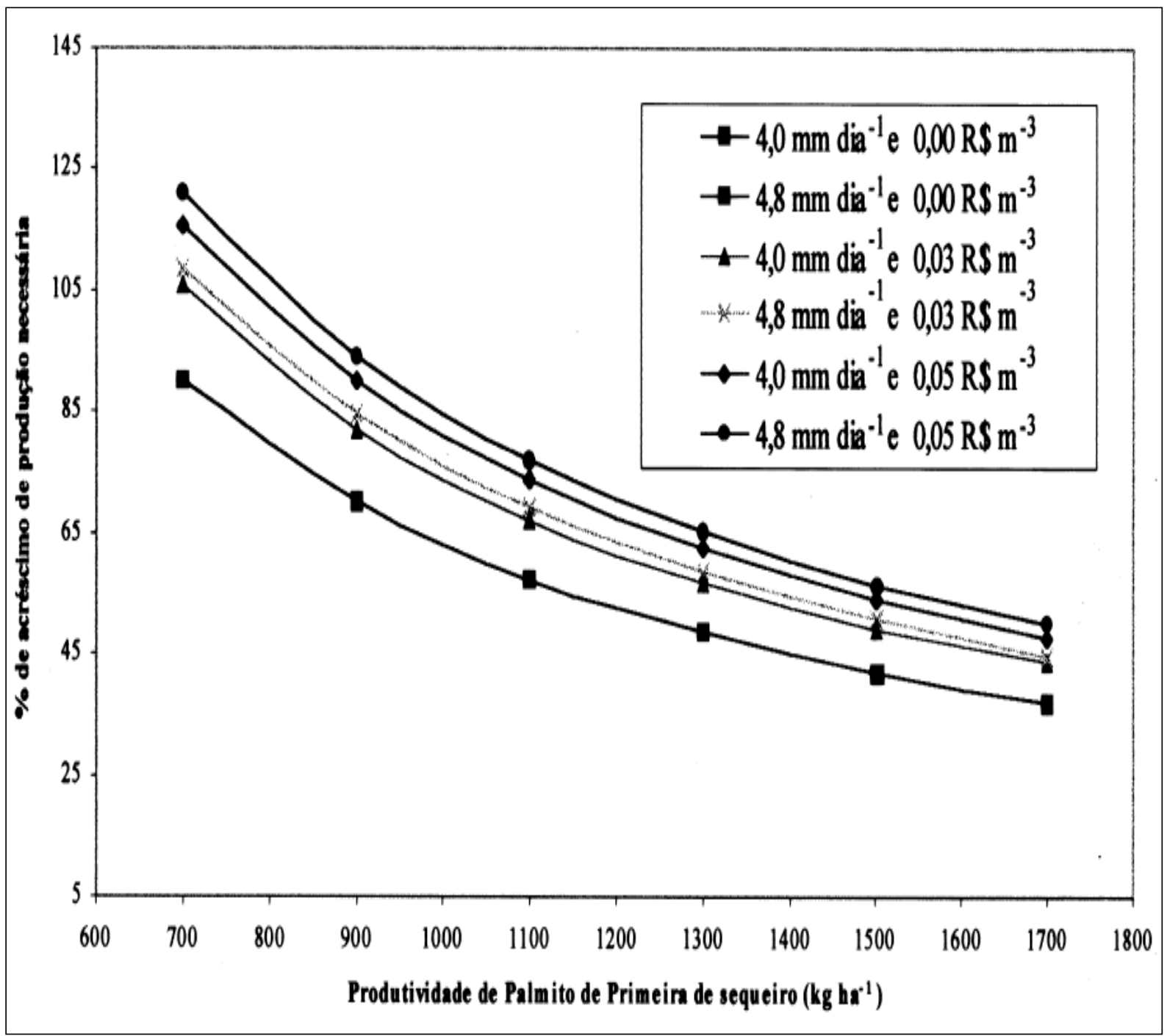

Figura 1 - "Acréscimos de produtividade necessário" para as combinações de três custos de água e evapotranspiração de 4 mm dia ${ }^{-1}$ e $4,8 \mathrm{~mm} \mathrm{dia}^{-1}$, considerando os demais valores fixos.

Ciência Rural, v. 33, n. 2, mar-abr, 2003. 


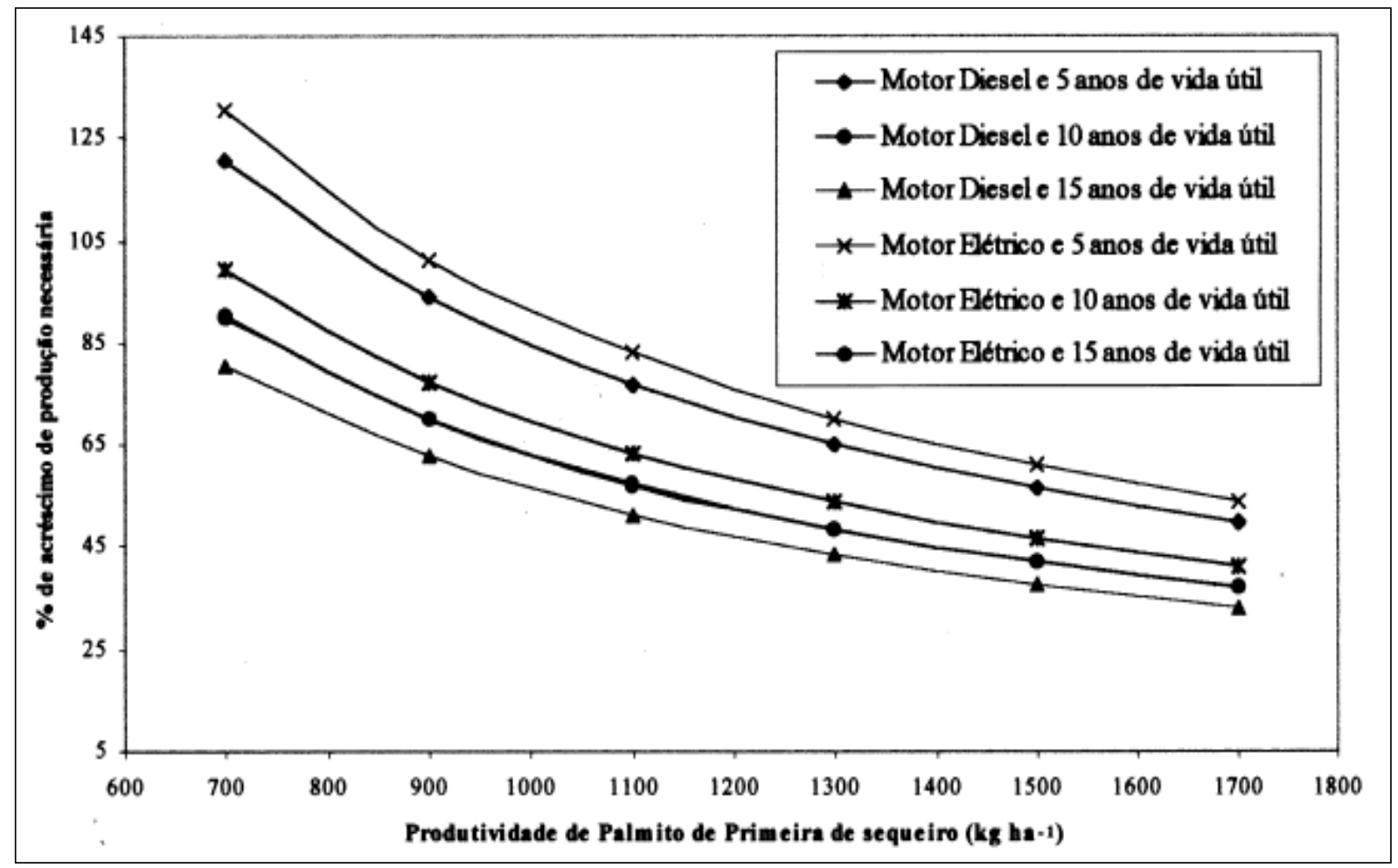

Figura 2 - "Acréscimo de produtividade necessário" para as combinações de Motor Diesel e Motor Elétrico considerando a vida útil do equipamento de 5 anos, 10 anos e 15 anos; e demais fatores fixos.

equivalente a $280,7 \mathrm{~kg} \mathrm{ha}^{-1}$. Comparando-se, para as condições da avaliação, o motor diesel com um diferencial máximo de "acréscimo de produtividade necessário" de $120,62 \%$ contra o motor elétrico com diferencial máximo de "acréscimo de produtividade necessário" de 130,33\%, tendo então o uso do motor diesel apresentado o melhor desempenho.

Considerando a produtividade da pupunheira irrigada para esta localidade, de $2500 \mathrm{~kg} \mathrm{ha}^{-1}$ e a produtividade de pupunheira não irrigada de $700 \mathrm{~kg} \mathrm{ha}^{-1}$ (LOPES et al., $2000 \mathrm{e}$ HERNANDEZ, 2001b), observou-se um acréscimo de produtividade $257 \%$. Analisando-se as simulações realizadas, observou-se que na primeira simulação, o maior "acréscimo de produtividade necessário" foi de $121,08 \%$ para produtividade de sequeiro de $700 \mathrm{~kg} \mathrm{ha}^{-1}, 80 \%$ de lucratividade, aplicação de 4,8 $\mathrm{mm} \mathrm{dia}^{-1}$, custo da água de $0,05 \mathrm{R} \$ \mathrm{~m}^{-}$ ${ }^{3}$ e motor elétrico; já para a segunda simulação o máximo valor de "acréscimo de produtividade necessário" foi de $130,33 \%$ para produtividade de sequeiro de $700 \mathrm{~kg} \mathrm{ha}^{-1}, 80 \%$ de lucratividade, aplicação de 4,0 $\mathrm{mm} \mathrm{dia}^{-1}$, custo da água de $0,00 \mathrm{R} \$ \mathrm{~m}^{-}$ ${ }^{3}$ e motor elétrico.

\section{CONCLUSÕES}

A irrigação da pupunheira é viável para a localidade de Ilha Solteira - SP, pois o aumento de produtividade real de $257 \%$, ocorrido com a irrigação, supera o máximo "acréscimo de produtividade necessário” de 130,33\%.

\section{REFERÊNCIASBIBLIOGRÁFICAS}

AGRIANUAL. Pupunheira é alternativa real. In: Anuário estatístico da agricultura brasileira. São Paulo: FNP/M\&S, 1997. p.327-332. (Agrianual 98).

AZEVEDO, J.A. Aspectos sobre o manejo da irrigação por aspersão para o cerrado. Brasília: EMBRAPA/CPCA, 1983. 53p. EMBRAPA/CPAC. (Circular Técnica, 16).

BERNARDO, S. Manual de irrigação. 6.ed. Viçosa : UFV, 1995. 657p.

BOVI, M.L.A. Palmito pupunha - Informações básicas para o cultivo. Campinas : Instituto Agronômico, 1997. 11p. (Boletim Técnico, IAC).

CAMARGO, I.M.T. Noções básicas de engenharia econômica: aplicações ao setor elétrico. Brasília : FINATEC, 1998. 160p. 
CENTURION, J.F. Balanço hídrico da região de Ilha Solteira. Científica, São Paulo, v.10, n.1, p.57-61, 1982.

CLARK, E.; J ACOBSON, K.; OLSON, D.C. Avaliação econômica e financeira de projetos de irrigação. Brasília : Ministério da Integração Nacional/Secretaria da Irrigação, 1993. (Manual de irrigação, 3).

CLEMENT, C.R. Growth and genetic analysis of pejibaye (Bactris gasipaes Kunth, Palmae) in Hawaii. 1995. 221f. PhD Thesis - Dept Horticulture, University of Hawaii at Manoa.

CLEMENT, C.R. Pupunha (Bactris gasipaes Kunth, Palmae). Jaboticabal : Funep, 2000. 48p.

DEFRANK, J.; CLEMENT, C.R. Weed control in pejibaye heart-of-palm plantations in Hawaii. HortScience, v.30, n.6, p.1215-1216. 1995.

FERREIRA, S.A.N. A cultura da pupunheira. Revista Brasileira de Fruticultura, v.9, p.23-28, 1987.

FRANCISCO, W. Matemática financeira. 7ed. São Paulo: Atlas, 1991. 319p.

FRIZZONE, J.A.; BOTREL, T.A.; FREITAS, H.A.C. Análise comparativa dos custos de irrigação por pivô-central, em culturas de feijão, utilizando energia elétrica e óleo diesel. Engenharia Rural, Piracicaba, v.5, n.1, p.34-53, 1994.

FRIZZONE, J.A. Planejamento da irrigação uma abordagem às decisões de investimento. Piracicaba: ESALQ/USP, 1999. 110p.

HERNANDEZ, F.B.T; LEMOS FILHO, M.A.F; BUZETTI, S. Software hidrisa e o balanço hídrico de Ilha Solteira. Ilha Solteira : UNESP, 1995. 45p. (Manual do Usuário, Série Irrigação, 1).
HERNANDEZ, F.B.T. Irrigação na cultura da pupunheira no noroeste paulista. Capturado em 20 mai. 2001a. Online. Disponível na Internet http://www.agr.feis.unesp.br/ aracatuba.htm.

HERNANDEZ, F.B.T. ENCONTRO TÉCNICO SOBRE A CULTURA DA PUPUNHA E DO COCO DA BAÍA NA NOVA ALTA PAUlistA, 2., 2001. Anais... Capturado em 20 mai. 2001b. Online. Disponível na Internet http:// www.agr.feis.unesp.br/irrigapup_6.htm.

LOPES, A.S. et al. Manejo da irrigação na cultura da pupunha (Bactris gasipaes H.B.K.) no noroeste paulista. In: CONGRESSO BRASILEIRO DE ENGENHARIA AGRÍCOLA, 29., 2000, Fortaleza. Anais... Fortaleza : SBEA, 2000. Trabalho EAS248 (CD-ROM)

MAROUElli, W.A.; SILVA, W.L.C. Seleção de sistemas de irrigação para hortaliças. Brasília : EMBRAPA, 1998. 16p. (Circular Técnica da Embrapa Hortaliças, 11).

MARQUES, P.A.A.; MARQUES, T.A.; COELHO, R.D. Programa pupunha: software para avaliação econômica da irrigação da pupunha. In: CONGRESSO BRASILEIRO DE ENGEnharia AGRÍCOlA, 28., 1999, Pelotas. Anais... Pelotas : SBEA, 1999. Trabalho 112, (CD-ROM).

MORO, J.R. Produção de palmito de pupunha. Viçosa : Centro de Produções Técnicas, 1996. 28p. (Manual nº 87).

RAMOS, A. Desenvolvimento vegetativo da pupunheira (Bactris gasipae Kunth) irrigada por gotejamento em função de diferentes níveis de depleção de água no solo. 1998. 66f. Dissertação (Mestrado em Irrigação e Drenagem) - Escola Superior de Agricultura "Luiz de Queiroz", Universidade de São Paulo.

TONET, R.M.; FERREIRA, L.G.S.; OTOBONI, J.L.M. A cultura da pupunha. Campinas : Instituto Agronômico,1999. 44p. (Boletim Técnico IAC, 237). 